\title{
Microfluidic models of atherosclerosis: replacing animal testing with on-chip technology
}

\author{
Keywords: microfluidics, atherosclerosis, tissue engineering, or- \\ gan-on-chip
}

\section{Introduction}

Coronary artery disease (CAD) is the principal cause of morbidity and mortality worldwide. In the US alone, CAD affects $>16$ million adults, accounts for $>1 / 3$ of all deaths, and is responsible for $>1.2$ million hospitals annually. ${ }^{1}$ The pathogenesis of CAD is complex and multi factorial, and is initiated by the formation of atherosclerosis. This insidious process renders plaque accumulation in coronary arteries, may result in downstream ischemia of myocardial tissue, and culminates in myocardial infarction or sudden cardiac death. ${ }^{2}$ In vivo models of coronary atherosclerosis have been primarily in mice-due to their relative low cost and convenience in handling-despite a general physiologic dissimilarity to humans. Concerted effort has been made to develop in vitro models for patient-specific dosing of anticoagulants, but has been limited to genotyping and platelet aggregation assays that do not accurately simulate hemodynamic settings experienced by patients with CAD.

The concept of replacing animal models with human cells cultured in microfluidic devices, recently referred to as organ-onchip technology, ${ }^{3}$ is gaining traction but still very far from replacing animal models as the gold standard for predicting the efficacy of drugs in humans. The major hurdle is accumulating enough evidence to demonstrate that in vitro systems can accurately recapitulate aspects of human pathology. However, for atherosclerosis and many other diseases, many aspects of the pathology are still being elucidated, ${ }^{2}$ supporting the need for in vitro models that can rapidly be redesigned to investigate the latest findings. For example, current clinical practice correlates a smaller narrowing of a coronary artery with a poor prognosis for the patient. This clinical metric is based on the long held belief that activation of platelets is proportional to the maximal level of wall shear stress (WSS), which occurs at the smallest narrowing of a stenosis. Experimental evidence, however, suggests that the activation of platelets is better correlated to the duration of exposure to a sufficiently high level of shear, rather than the maximal level of WSS. ${ }^{4,5}$ This is particularly interesting considering that platelets typically only reside within a stenosis for $<0.1 \mathrm{~s}$ and have a low probability of re circulating to the same stenotic vessel. ${ }^{6}$ Therefore, the variance in duration of exposure is contributed to the disturbed flow profiles that occur within stenotic vessels. Often these conditions are not recapitulated in vitro since models do not include pulsatile flow and/or arterial capacitance, which significantly alter the fluid dynamics.

In addition to fluid dynamic parameters, platelet activation is strongly correlated with exposure to chemicals and proteins secreted by cells in the artery. ${ }^{6}$ While damage to endothelial cells has been historically believed to be correlated to maximal shear stress; most of these studies, however, were performed with continuous flow, which is not physiological. Experiments using physiologically-relevant pulsatile flow show, in fact, that low shear levels induce greater production of pro-coagulant proteins, such as tissue factor. ${ }^{6}$ These
Volume 2 Issue 4 - 2017

\author{
Bobak Mosadegh \\ Department of Radiology, New York Presbyterian Hospital \& \\ Weill Cornell Medicine, USA
}

Correspondence: Bobak Mosadegh, Department of Radiology, New York Presbyterian Hospital \& Weill Cornell Medicine, 413 E69th St. Suite 108, New York, USA, Tel 21 2-327-7| 87, Email bom2008@med.cornell.edu

Received: June 0I, 2017 | Published: June 06, 2017

results reconcile the in vivo observations that plaque formation occurs in regions of low shear and disturbed flow. Using computational fluid dynamics (CFD), factors associated with platelet activation (i.e., shear stress and residence time) further elucidate our understanding of where and why plaque accumulates in certain locations. What is not understood, however, is when a plaque will rupture, causing sudden occlusion of the artery, an event believed to account for more than half of all deaths associated with CAD. There is little correlation between the size of the stenosis and the probability of plaque rupture. ${ }^{7}$ Therefore, a significant effort to diagnose the vulnerability of plaques and even prevent its rupture is being extensively explored. Unfortunately, as described above, there are no proper models that allow for efficient and predictive validation of new methods.

\section{Conclusion}

There is a need to develop microfluidic models of atherosclerosis that recapitulate each of the different stages of the disease, and categorized by whether the goal of the device is to screen pharmaceutical drugs, elucidate a biological mechanism, or perform a diagnostic. In general, there are three uses for such a microfluidic device will be described as examples of the broad applicability and impact that this technology can have in advancing our knowledge and developing treatments for this devastating disease.

The first use would serve as a means to provide personalized medicine by optimizing the dosage of a blood thinner to a patient. There will be two aspects that would make such a device patientspecific, one is the use of the patient's own blood in the device, and the second is the device would be tailored to match the patient's fluid dynamic properties at the stenosis. Boundary conditions specific to the patient's stenosis could be acquired using CT imaging and subsequent CFD analysis. By determining which combination of drugs and at what concentrations is optimal to avoid undesired clotting and bleeding, patients could forego the unnecessary complications associated pharmaceutical treatment.

The second use of such a device would be to recapitulate the early stages of the disease, when an atheroma is beginning to form. This platform would allow testing of drugs that prevent the rupture of the plaque by thickening its fibrous cap. Methods to achieve this is a 
controversial issue since most approaches to thicken the cap will also promote growth of the plaque itself, ${ }^{2}$ which may lead to downstream ischemia of the myocardial tissue. Such methods include, promoting vascularization of the plaque via the vasa vasorum to reduce the size of the necrotic core, promoting migration of SMCs toward the intima to reinforce the fibrous cap, or promoting junctions between endothelial cells. The device should consist of endothelial cells, vascular smoothmuscle cells, and monocytes. A high LDL-containing medium will be exposed to the cells at various shear stresses and with different combination of drugs to determine potential candidates, based on the prevention of the size and morphology of the plaque.

The third use of this device will recapitulate the plaque rupture itself as an acute event to test drugs that can actively prevent the coagulation process that causes thrombi to form rapidly and occlude the artery. This device could mechanically induce a rupture of the plaque, using integrated pneumatic actuators, in the presence of flowing blood so that an acute thrombosis would form. Since the strain on the fibrous cap is achieved actively, the timing, size and site of the rupture would be reproducible allowing for statistically relevant data to be acquired for determining the effect of various drugs. Furthermore, the plaque itself, in its final disease state, would be directly fabricated to allow different geometries and sizes of the plaques to be assessed.

By developing these classes of microfluidic devices, the treatment of atherosclerosis can be enhanced by rapidly testing the nextgeneration drugs that combat various stages of this chronic disease. Furthermore, these devices will provide a platform for researchers to conduct mechanistic studies and develop novel imaging tools to determine the vulnerability of a plaque.

\section{Acknowledgements}

Bobak Mosadegh was supported by the National Center for Advancing Translational Sciences of the National Institutes of Health under Award Number UL1TR000457.

\section{Conflict of interest}

The author declares no conflict of interest.

\section{References}

1. Go AS, Mozaffarian D, Roger VL, Benjamin EJ, et al. Heart disease and stroke statistics--2014 update: a report from the American Heart Association. Circulation. 2014;129(3):e28-292.

2. Badimon L, Vilahur G. Thrombosis formation on atherosclerotic lesions and plaque rupture. $J$ Intern Med. 2014;276(6):618-632.

3. Huh D, Torisawa YS, Hamilton GA, et al. Microengineered physiological biomimicry: Organs-on-Chips. Lab Chip. 2012;12(12):2156-2164.

4. Rubenstein DA, Yin W. Quantifying the effects of shear stress and shear exposure duration regulation on flow induced platelet activation and aggregation. J Thromb Thrombolysis. 2010;30(1):36-45.

5. Jesty J, Yin W, Perrotta P, et al. Platelet activation in a circulating flow loop: combined effects of shear stress and exposure time. Platelets. 2003;14(3):143-149.

6. Yin W, Shanmugavelayudam SK, Rubenstein DA. The effect of physiologically relevant dynamic shear stress on platelet and endothelial cell activation. Thromb Res. 2011;127(3):235-241.

7. Sannino A, Brevetti L, Giugliano G, et al. Non-invasive vulnerable plaque imaging: how do we know that treatment works? Eur Heart J Cardiovasc Imaging. 2014;15(11):1194-1202. 